\title{
Concentration of circulating miRNA-containing particles in serum enhances miRNA detection and reflects CRC tissue- related deregulations
}

\author{
Abdou EISharawy ${ }^{1,6}$, Christian Röder ${ }^{2}$, Thomas Becker ${ }^{3}$, Jens K. Habermann ${ }^{4}$, \\ Stefan Schreiber ${ }^{1,5}$, Philip Rosenstiel ${ }^{1}$, Holger Kalthoff ${ }^{2}$ \\ ${ }^{1}$ Institute of Clinical Molecular Biology, Christian-Albrechts-University, Kiel, Germany \\ ${ }^{2}$ Institute for Experimental Cancer Research, Christian-Albrechts-University, Kiel, Germany \\ ${ }^{3}$ Department of General Surgery, Visceral, Thoracic, Transplantation and Pediatric Surgery, University Hospital Schleswig- \\ Holstein, Campus Kiel, Kiel, Germany \\ ${ }^{4}$ Section for Translational Surgical Oncology and Biobanking, Department of Surgery, University of Lübeck and University \\ Hospital Schleswig-Holstein, Campus Lübeck, Lübeck, Germany \\ ${ }^{5}$ Clinic for Internal Medicine I, University Hospital Schleswig-Holstein, Campus Kiel, Kiel, Germany \\ ${ }^{6}$ Faculty of Sciences, Division of Biochemistry, Department of Chemistry, Damietta University, New Damietta City, Egypt \\ Correspondence to: Abdou Elsharawy, email: a.sharawy@mucosa.de
}

Keywords: serum biomarker, extracellular microvesicles, miRNA-containing particles, colorectal neoplasms and inflammatory bowel disease, strand-specific miRNA deregulation

Received: February 08, 2016

Accepted: September 12, 2016

Published: September 23, 2016

\section{ABSTRACT}

The emerging potential of miRNAs as biomarkers for cancer detection demands parallel evaluation of strategies for reliable identification of disease-related signatures from easily accessible and pertinent body compartments. Here, we addressed whether efficient concentration of circulating miRNA-carrying particles is a rationale for miRNA biomarker discovery. We systematically compared miRNA signatures in 93 RNA preparations from three serum entities (whole serum, particle-concentrated, and particle-depleted fractions) and corresponding tissue samples from patients with colorectal cancer (CRC) as a model disease. Significant differences between whole sera and particle-concentrated serum fractions of CRC patients emerged for 45 of $\mathbf{7 4 2}$ tested miRNAs. Twenty-eight of these $\mathbf{4 5}$ miRNAs were differentially expressed between particle-concentrated serum fractions of metastatic CRC- and healthy individuals. Over half of these candidates (15 of 28 ) showed deregulations only in concentrated serum fractions, but not in whole sera, compared to the respective controls.

Our results also provided evidence of a consistent downregulation of miR-486 and miR-92a, and further showed a possible "strand-specific" deregulation of extracellular miRNAs in CRC. More importantly, most of the identified miRNAs in the enriched sera reflected the patterns of the corresponding tumor tissues and showed links to cancerrelated inflammation. Further investigation of seven serum pools revealed a subset of potential extracellular miRNA candidates to be implicated in both neoplastic and inflammatory bowel disease.

Our findings demonstrate that enrichment and sensitive detection of miRNA carriers is a promising approach to detect CRC-related pathological changes in liquid biopsies, and has potential for clinical diagnostics.

\section{INTRODUCTION}

It is becoming increasingly clear that circulating miRNAs represent suitable biomarkers for a broad spectrum of diseases [1, 2], e.g. for cancer. In addition, they are important mediators of cell-cell communications [3]. This class of small noncoding RNAs controls gene expression by different mechanisms and, when 
released by cancer cells, may induce a pro-metastatic inflammatory response [4]. A major challenge is the establishment of a sensitive, specific and robust detection strategy of disease-related miRNA signatures in patient's blood samples.

Several recent studies characterized circulating miRNAs as protein-bound $[5,6]$ and utilized suitable immunoprecipitation protocols for isolation of such ribonucleo-proteins [7]. Other studies demonstrated that miRNAs are contained and transported in extracellular microvesicles (EVs) or exosomes present in biological fluids $[8,9]$. The key arguments for considering EV miRNAs in biomarker discovery are that (i) these EVs have complex biochemical structures that protect their contents and resemble the cells they originated from; (ii) profiling EVs cargo (RNA, proteins, etc.) can be a window to the genetic status of individual tissues, providing important biological/functional insights, and can be regarded as a fingerprint of a disease; and (iii) understanding these mechanisms in this new area of research is of great diagnostic, prognostic and therapeutic potential for human diseases [10-12].

Many reports also investigated whole blood as an easily accessible compartment for detection of extracellular miRNAs $[1,13,14]$. Yet, other studies have argued against this because of the interferences of blood cell-miRNAs and possible hemolysis as an additional source of unwanted miRNA [15]. Moreover, the actual origin and function of extracellular miRNAs, as either "non-specific by-products" or "on-purpose messengers", remain under debate [16].

These studies demonstrate the challenges of investigating miRNA (signatures) and might explain why other studies analyzed miRNAs in the tissue of interest directly, rather than extracellular miRNA [17]. Yet, noninvasive early detection, through standardized (pre) analytical procedure with a focus on EV-miRNAs, is currently needed [18].

In the light of this information, together with the heterogeneity of the reported studies (analysis of whole blood, serum/plasma, fractions thereof, or tissue samples; detection by microarray, qPCR, or sequencing; data normalization; etc), it is not surprising that little overlap has emerged between the results of different biomarker studies for the same disease.

These observations reflect the need to evaluate suitable strategies for reliable identification of miRNA disease-related signatures from easily accessible biofluids. We therefore followed a comprehensive approach, which integrates an efficient method for isolation of the "bulk" of miRNA carriers prior to sensitive qPCR profiling, to compare miRNA expression patterns in three serum entities (whole serum, particleconcentrated, and particle-depleted fractions), and tissue samples from the same patients with colorectal cancer (CRC) as a model disease.

\section{RESULTS}

\section{Discovery of miRNA blood biomarker candidates for CRC in whole sera compared to particle-concentrated serum fractions}

The strategy of our study to identify CRC-related miRNA biomarkers in the blood is depicted in Figure 1. A general focus was laid on the possible impact of a robust and easy-to-perform pre-analytic concentration of miRNA-carrying particles in favor of an improved detection of CRC-specific miRNA signatures in the blood. Comparison of the detectability of 742 miRNAs in 30 RNA preparations from different serum fractions (Figure 1) of 10 CRC patients (UICC stage IV, compare Supplementary Table S2a) was performed on Exiqon miRCURY LNA ${ }^{T M}$ Universal RT miRNA PCR Human panels-I-and-II and revealed substantial differences depending on the pre-analytic processing of the sera. The total number of detected miRNAs was higher in the particle-concentrated fractions, than in the whole sera and corresponding particle-depleted fractions ( $\sim 150-250, \sim 80$ 120 , and $\sim 20-50$ miRNAs, respectively) (Supplementary Figure S1).

Following PCR-panel processing, normalization and filtering, $>40$ miRNAs were detected on average in all whole sera and particle-concentrated fractions, but only 11 miRNAs in the particle-depleted fractions. Statistical analyses showed a separation and clustering of the matched particle-concentrated and supernatant fractions (Supplementary Figures S2 and S3). Each of two normalization approaches employed (Supplementary Data), yielded 44 statistically significant miRNA candidates, which showed differential expression between whole sera and the corresponding particle-concentrated fractions (Supplementary Table S1). Of all miRNAs analyzed, 43 candidates with consistent detectability after both normalizations, plus two unique miRNAs (miR-143, miR-423), were considered for the subsequent analysis.

In addition, we assessed the effect of different RNA preservation methods for tissue miRNA detection (details in Supplementary Data, Supplementary Tables S5 and S6, and Supplementary Figure S4). Accordingly, we considered only the data of the tissues preserved in liquid nitrogen $(n=15)$ in our downstream analyses in order to avoid additional confounding factors.

\section{Improved detection of miRNA biomarker candidates after enrichment of miRNA carriers from serum}

Further analysis and comparison of expression of the selected 45 miRNA candidates in 63 independent RNA preparations, from serum and corresponding tumor tissue samples (Supplementary Table S2b; Figure 1), resulted in 22 differentially expressed miRNAs in patients' particle- 
concentrated sera (Table 1; Supplementary Table S3), but only 13 miRNAs in patients' whole sera (Supplementary Table S4).

We observed an overlap of 12 detected miRNAs between whole sera and the particle-concentrated fraction. However, 10 miRNAs were only identified in the particleconcentrated fractions (Figure 2). Interestingly, one miRNA (miR-26a) showed elevated expression only in the whole sera. Yet, miR-26a was also emerged in the particleconcentrated fractions when comparing enriched serum fractions of metastatic CRC patients to those of controls (Figure 2c).

\section{miRNA expression in particle-concentrated sera mirrors deregulation of tumor tissues}

To evaluate whether miRNA patterns in the particleconcentrated fractions reflect deregulations of miRNA expression in the corresponding tumors, we examined the detectability of the 45 miRNA candidates (I) in the particleconcentrated sera of patients versus controls, (II) in the particle-concentrated sera of CRC patients versus their corresponding tumor tissues, and (III), in these CRC tissues versus particle-concentrated sera of control individuals. The twenty two miRNA candidates with differential expression in patients' particle-concentrated sera are listed in Table 1 . The detection of more than two-third (15/22) of these miRNAs was consistent in CRC-patients' particle-concentrated sera and the matched tumor tissue samples (indicated by "Yes" in Table 1/columns 7 and 10). Ten of these 15 miRNAs were simultaneously overexpressed, whereas five showed consistently decreased expression (marked grey in Table $1)$. Five of the 10 overexpressed miRNAs (miR-29c$3 p,-23 a-3 p,-23 b-3 p,-24-3 p$, and $-125 b-5 p)$ showed an elevated expression in tissues than in the matched particleconcentrated sera. Two of these five miRNAs (miR23a-3p, miR-125b-5p) were exclusively identified in the particle-concentrated compartment (Table 1). Similarly, four of the five miRNAs with reduced expression, in both CRC-patients' particle-concentrated sera and the matched tumor tissue samples (i.e., all except for miR-486-5p), were exclusively identified in CRC-patients' particleconcentrated sera, but not in the whole serum samples. However, the remaining miRNAs (7/22; Table 1) showed

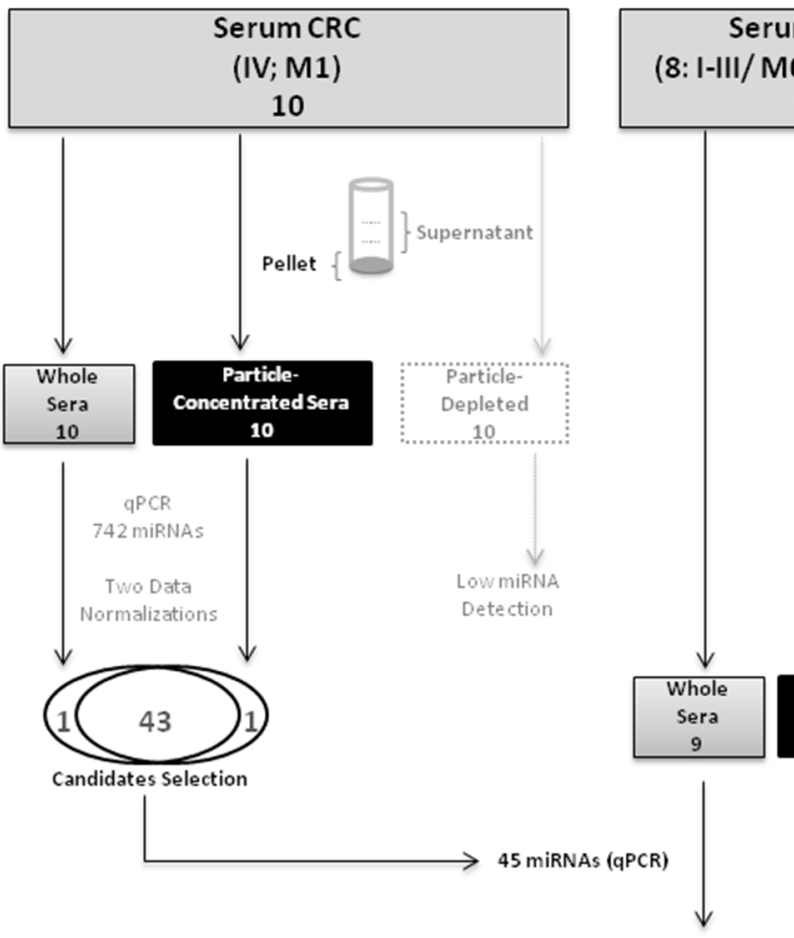

Serum CRC
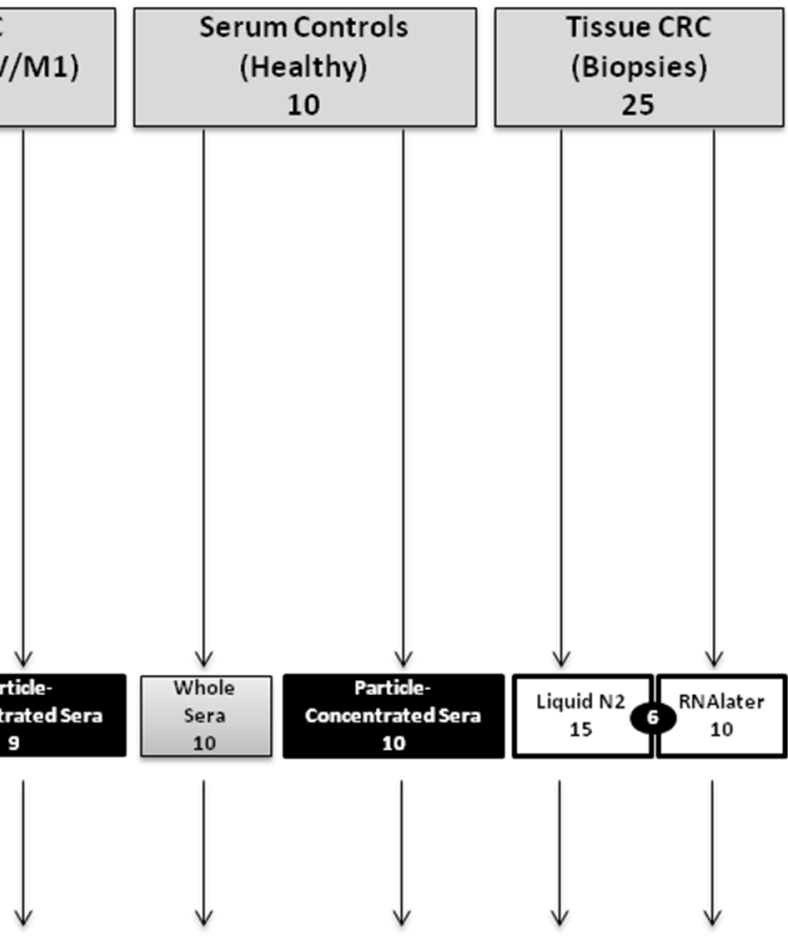

Comparison of the expression patterns of the selected 45 miRNAs in all tested patient and control samples

Independent investigation of extracellular miRNAs in seven (nuclease-pretreated) serum pools (CRC, UC, and control)

Figure 1: Study design and experimental setup. This figure shows the types of the 93 RNA samples tested in this study. Using a 742-miRNA qPCR panel, we first identified 45 differentially expressed miRNAs, which we further investigated in 63 RNA samples from an independent CRC patients' and healthy controls' sera as well as paired tumor tissue samples. We additionally analyzed extracellular miRNA patterns, using another EVs isolation method, in seven (nuclease-pretreated) serum pools of colorectal cancer (CRC), ulcerative colitis (UC) and healthy individuals. 
Table 1: Particle-Concentrated serum miRNA patterns reflect CRC tissue-related expression deregulations

\begin{tabular}{|c|c|c|c|c|c|c|c|c|c|}
\hline \multirow[b]{2}{*}{$\begin{array}{l}\text { Expression } \\
\text { pattern with } \\
\text { respect to } \\
\text { CRC }\end{array}$} & \multirow[b]{2}{*}{$\begin{array}{c}\text { miRNA } \\
\text { candidates }\end{array}$} & \multicolumn{2}{|c|}{$\begin{array}{c}\text { I. Particle- } \\
\text { concentrated sera } \\
\text { (CRC vs. controls) }\end{array}$} & \multicolumn{2}{|c|}{$\begin{array}{c}\text { II. Particle- } \\
\text { concentrated } \\
\text { sera vs. tissue (all } \\
\text { CRC) }\end{array}$} & \multirow{2}{*}{$\begin{array}{c}\text { Consistent } \\
\text { expression } \\
\text { of I \& II } \\
(15 / 22 ; \\
68 \%)\end{array}$} & \multicolumn{2}{|c|}{$\begin{array}{l}\text { III. Tissue (CRC) } \\
\text { vs. particle- } \\
\text { concentrated sera } \\
\text { (controls) }\end{array}$} & \multirow[t]{2}{*}{$\begin{array}{c}\text { Consistent } \\
\text { expression } \\
\text { of I \& III } \\
(20 / 22 ; \\
91 \%)\end{array}$} \\
\hline & & $\begin{array}{c}\text { Fold } \\
\text { change }^{\mathrm{a}}\end{array}$ & $\begin{array}{l}\text { Adjusted } \\
\text { P-value }\end{array}$ & $\begin{array}{c}\text { Fold } \\
\text { change }^{b}\end{array}$ & $\begin{array}{l}\text { Adjusted } \\
\text { P-value }\end{array}$ & & $\begin{array}{c}\text { Fold } \\
\text { change }^{c}\end{array}$ & $\begin{array}{l}\text { Adjusted } \\
\text { P-value }\end{array}$ & \\
\hline \multirow{10}{*}{$\begin{array}{l}\uparrow \uparrow \\
\text { Particle- } \\
\text { concentrated } \\
\text { Sera \& tissue } \\
(n=10)\end{array}$} & miR-22-3p & 2.673 & $3.04 \times 10^{-05}$ & 0.311 & 0.4852 & Yes & 2.362 & $2.02 \times 10^{-06}$ & Yes \\
\hline & $\operatorname{miR}-21-5 p$ & 4.766 & $5.65 \times 10^{-05}$ & -1.123 & 0.2398 & Yes & 5.889 & $7.05 \times 10^{-07}$ & Yes \\
\hline & $\operatorname{miR}-29 c-3 p$ & 2.482 & $5.65 \times 10^{-05}$ & -1.738 & 0.0059 & Yes & 4.221 & $7.53 \times 10^{-08}$ & Yes \\
\hline & miR-101-3p & 3.334 & $8.91 \times 10^{-05}$ & 1.345 & 0.1126 & Yes & 1.989 & 0.0207 & Yes \\
\hline & miR-23a-3p & 0.673 & 0.0002 & -0.882 & 0.0005 & Yes & 1.555 & $2.62 \times 10^{-07}$ & Yes \\
\hline & $\operatorname{miR}-23 b-3 p$ & 1.259 & 0.0004 & -2.766 & $1.16 \times 10^{-08}$ & Yes & 4.026 & $4.60 \times 10^{-12}$ & Yes \\
\hline & miR-423-5p & 2.516 & 0.0010 & 1.053 & 0.1126 & Yes & 1.463 & 0.0018 & Yes \\
\hline & miR-24-3p & 1.473 & 0.00293 & -1.684 & 0.0019 & Yes & 3.157 & $1.66 \times 10^{-09}$ & Yes \\
\hline & let-7f-5p & 1.328 & 0.0095 & -1.065 & 0.1326 & Yes & 2.394 & 0.0022 & Yes \\
\hline & miR-125b-5p & 1.243 & 0.0454 & -5.460 & $5.17 \times 10^{-08}$ & Yes & 6.704 & $8.05 \times 10^{-13}$ & Yes \\
\hline \multirow{3}{*}{$\begin{array}{l}\uparrow \text { Particle- } \\
\text { concentrated } \\
\text { sera only } \\
(n=3)\end{array}$} & $\operatorname{miR}-22-5 p$ & 6.130 & $7.40 \times 10^{-05}$ & 5.360 & 0.0001 & No & 0.770 & 0.1898 & Yes \\
\hline & $\operatorname{miR}-223-3 p$ & 2.151 & 0.0051 & 2.292 & 0.0083 & No & -0.140 & 0.8114 & Yes \\
\hline & miR-320b & 0.795 & 0.0134 & 1.103 & 0.0030 & No & -0.307 & 0.3353 & Yes \\
\hline \multirow{2}{*}{$\begin{array}{l}\uparrow \text { Particle- } \\
\text { concentrated } \\
\text { sera \& } \\
\downarrow \text { tissue }(n=2)\end{array}$} & $\operatorname{miR}-335-5 p$ & 1.832 & 0.0002 & 3.241 & $2.48 \times 10^{-09}$ & No & -1.408 & 0.0024 & No \\
\hline & miR-144-3p & 3.330 & 0.0004 & 6.713 & $6.45 \times 10^{-07}$ & No & -3.3820 & 0.0019 & No \\
\hline \multirow{5}{*}{$\begin{array}{l}\downarrow \downarrow \text { Particle- } \\
\text { concentrated } \\
\text { sera \& tissue } \\
(n=5)\end{array}$} & miR-486-5p & -2.119 & 0.0001 & 7.347 & $1.14 \times 10^{-14}$ & Yes & -9.466 & $3.35 \times 10^{-16}$ & Yes \\
\hline & miR-93-5p & -0.951 & 0.0003 & 0.489 & 0.0637 & Yes & -1.441 & $5.70 \times 10^{-07}$ & Yes \\
\hline & miR-92a-3p & -1.1269 & 0.0026 & -0.023 & 0.9485 & Yes & -1.103 & 0.0085 & Yes \\
\hline & $\operatorname{miR}-146 a-5 p$ & -0.777 & 0.0030 & 0.492 & 0.1820 & Yes & -1.270 & 0.0009 & Yes \\
\hline & miR-221-3p & -1.325 & 0.0064 & -0.057 & 0.9485 & Yes & -1.268 & 0.0036 & Yes \\
\hline \multirow{2}{*}{$\begin{array}{l}\downarrow \text { Particle- } \\
\text { concentrated } \\
\text { sera only } \\
(n=2)\end{array}$} & let-7d-3p & -2.006 & 0.00070 & -1.549 & 0.0049 & No & -0.456 & 0.1984 & Yes \\
\hline & $\operatorname{miR}-342-3 p$ & -1.205 & 0.0064 & -1.527 & 0.0003 & No & 0.321 & 0.3828 & Yes \\
\hline
\end{tabular}

$\uparrow$ : overexpression; $\downarrow$ : lower expression; ${ }^{\text {a }}$ : Fold changes $\left(\mathrm{ddC}_{\mathrm{q}}\right.$ values of particle-concentrated CRC sera minus those of the controls); positive values: upregulation in particle-concentrated CRC sera;

b: Fold changes ( $\mathrm{ddC}_{\mathrm{q}}$ values of particle-concentrated $\mathrm{CRC}$ sera minus those of the CRC tissue); positive values: upregulation in the particle-concentrated CRC sera or downregulations in the CRC tissue; ${ }^{c}$ : Fold changes (ddC ${ }_{q}$ values of CRC tissue minus those of the particle-concentrated sera of controls); positive values: upregulation in the CRC tissue. More details can be found in Supplementary Data (pp. 3-4). 
inconsistent expression between the particle-concentrated fractions and corresponding tissues (Supplementary Data). The ANOVA analysis underlined potential candidates with coordinated expressional changes of particle-concentrated sera and tissue miRNAs (Figure 3; Supplementary Table S7).

\section{miRNA in silico analyses and relation to cancer and inflammatory pathways}

In silico analyses of the identified 22 miRNAs (Table 1), using the miEAA tool, showed significant overrepresentation in the context of exosomes (17 miRNAs), and many diseases, such as carcinoma/adenocarcinoma (22 and 17 miRNAs), neoplasm/metastasis (21 miRNAs), inflammation (16 miRNAs), and colonic-neoplasms (10 miRNAs). Using other analysis options of miEAA, designated "OrgansmiRWalk" and "pathway-miRWalk", numerous significant overrepresentations of the identified 22 miRNAs in different organs/tissues and inflammation-/cancer-related pathways were found (Supplementary Table S8; Supplementary Data). A review of the current literature further confirmed the potential interaction of at least 16 of these 22 miRNAs (73\%; Supplementary Table S9) with key inflammatory-/cancerrelated factors, e.g., IL6, STAT3 and NF-кB.

\section{Extracellular miRNA candidates of pathophysiological relevance in metastatic CRC}

To address whether miRNA signatures in particleconcentrated serum fractions associate with CRC progression, we compared the expression pattern of the tested 45 miRNAs in patients with distant metastasis (M1) and individuals without metastasis (M0) to those of the controls. While 26 differentially expressed miRNAs were identified in the first comparison (CRC-M1 vs. controls), only 2 miRNAs (miR-335, miR-92a) were emerged in the second comparison (CRC-M0 vs. controls). These two miRNAs were also detected in the first 26-miRNA panel (Figure 2). Notably, 20 of the 22 miRNAs identified in the particle-concentrated sera were from these 26 miRNAs. Here, five of the six non-overlapping miRNAs between the two comparisons (Figure 2) would have been missed if we would have only analyzed whole sera. Altogether, these results underline that an additional 15 miRNAs ( $>53 \%$ ) were identified from the patients' particle-concentrated sera. Moreover, the expression data of these 26 miRNAs revealed similar coordinated patterns of expression in CRC-M1 particle-concentrated sera and paired tissue samples, with a previously reported link to inflammatory-/cancer-related factors (Supplementary Tables S10 and S11).

\section{Consistent expression patterns of 14 extracellular miRNAs in both CRC and IBD}

In order to explore which extracellular miRNAs are interrelated in their expression from the clinical samples of patients with CRC and inflammatory bowel disease (IBD), we also investigated miRNA patterns of EVs isolated from pooled sera of both CRC and ulcerative colitis (UC-)patients (six patients per pool). Seven serum pools (3 pools each for $\mathrm{CRC}$ and $\mathrm{UC}$ and one pool from healthy individuals) were
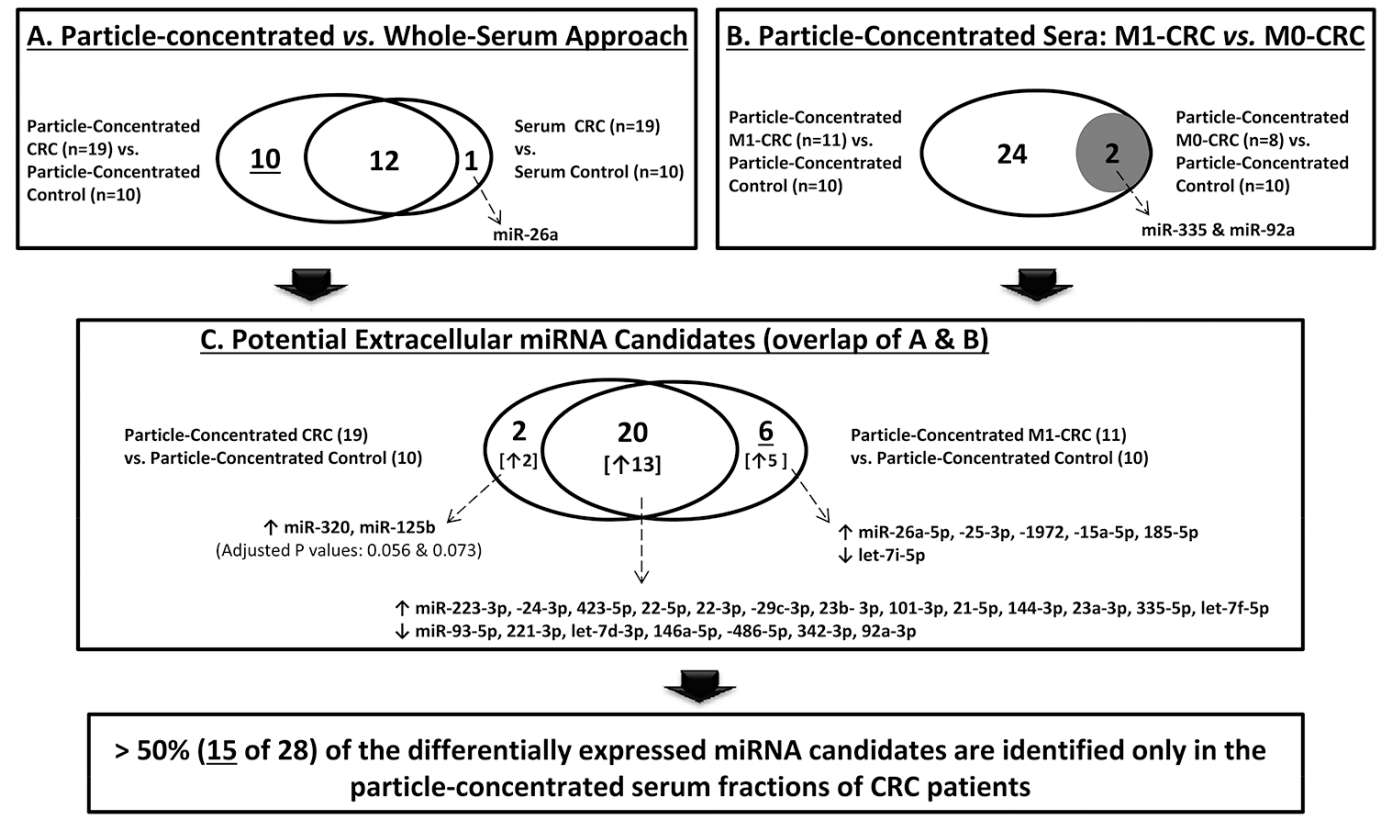

Figure 2: Differentially expressed extracellular miRNAs in CRC. This figure illustrates the different comparisons performed to identify differentially expressed miRNAs in CRC. Remarkably, more than half, 15 of 28 miRNA candidates (10 from A and 5 from C, after excluding miR-26a that was identified in the whole sera), were exclusively identified in CRC particle-concentrated serum compartment. ( $\uparrow$ : higher expression; $\downarrow$ : lower expression). 
pretreated with RNase and DNase and screened utilizing an Exiqon miRCURY LNA ${ }^{T M}$ Universal RT miRNA PCR Human panel I comprising 372 miRNA assays. Out of all 77 miRNAs detectable in all disease pools, only miR-30b was significantly differentially expressed $(\mathrm{P}=0.012)$ between CRC and UC pools. miR-30b was overexpressed in CRC compared to UC and healthy control pools. Interestingly, 14 miRNAs out of these 77 miRNAs showed differential expression of more than 2-fold in the comparisons of both CRC vs. healthy and UC vs. healthy pools. As shown in Figure 4, these 14 miRNAs were consistent in their expression between the two disease states compared to the healthy control pool. Notably, two of these miRNAs, miR$125 b-5 p$ (with higher expression) and miR-146a-5p (with lower expression) showed similar expression tendencies, as previously shown in our matched CRC serum and tissue samples (Table 1).

\section{DISCUSSION}

In the present study, we demonstrated for the first time that the concentration of circulating (EVs and non-
EVs) miRNA carriers based on steric exclusion, when followed by qPCR profiling, allowed a more reliable identification of CRC-related miRNAs. This approach further enabled the disclosure of candidates with potential functional relevance and allowed a better reflection of disease tissue-related patterns.

\section{Towards a comprehensive analysis of extracellular miRNAs}

The use of miRNAs as biomarkers in liquid biopsies is rapidly evolving. Yet, intra-and inter-experimental consistency and routine laboratory standards remain challenging [19]. Additionally, blood poses heterogeneous and poorly defined miRNA carriers and EVs composition [20]. Moreover, the biological source of extracellular miRNAs [12, 21] and EVs-cargo contents [22, 23] are currently under debate.

Here, we addressed some of these challenges at different levels of analysis. First, we utilized the particle precipitation method, which is time-saving, suited for clinical laboratories, and yielded higher RNA quantities

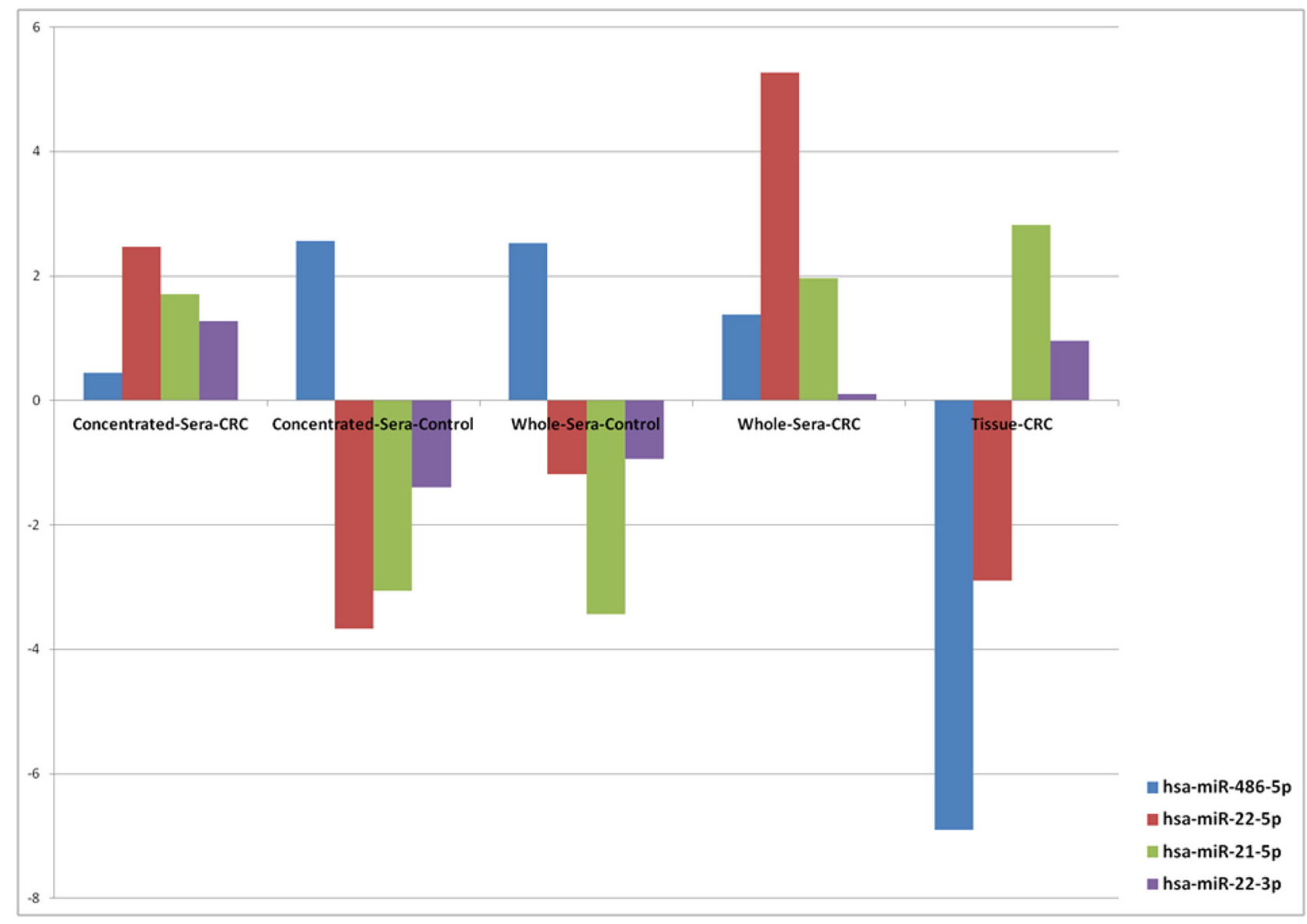

Figure 3: Expression trends of miRNAs in serum and CRC tissue samples. This figure shows examples of the results of ANOVA analysis of four miRNA candidates across five different sample types. Common low expression of miR-486-5p $\left(\mathrm{P}-\mathrm{value}, 3.39 \times 10^{-32}\right)$ and upregulation of miR-21-5p (P-value, $1.64 \times 10^{-10}$ ) were observed in CRC. A "strand-specific" expression was observed for miR-22. 
compared to other methods [24]. It precipitated the bulk of circulating miRNA populations, vesicle-trapped and protein-bound $[5,6,25]$. This co-precipitation maximizes the opportunity of permissible disclosure of disease-related markers. Yet, this isolation method is not appropriate to investigate specific miRNA carrier subpopulations. The intensive co-isolation of non-EVs complexes may, for instance, hamper and mask the detection of specific EVs miRNA carriers [26, 27]. Currently, there is an uncertainty in the biological/functional consequences of different particle compartments (EVs- and non-EVs). Also, the mechanism of extracellular RNA (as a "disposal" mechanism of unwanted/debris materials and/or a "control/ signaling" mechanism to alter other cells at a distance) remains unclear. Moreover, recent studies identified $\mathrm{AGO} 2$ in EVs [23], which has been primarily found in non-EVs-protein complexes, and has further demonstrated its potential function in sorting and secretion of specific miRNAs into EVs, in a KRAS-MEK-dependent manner [26]. These recent findings may suggest that EVs- and non-EVs miRNA carriers are somehow overlapping and cannot be fully regarded as two independent compartments of specific miRNAs, as traditionally thought $[5,6,28]$. Nevertheless, for functional analyses of specific miRNAs other established protocols can be utilized [7, 27].

Secondly, the correlation of miRNA patterns in serum of tumor patients with the corresponding tumor tissues can provide indications of the possible origins of the observed circulating miRNAs. This does not exclude miRNAs that are also present in normal colon tissues or activated by indirect effects of the tumor. Thirdly, only serum samples without hemolysis were considered for analysis to minimize the influence of blood-based miRNA patterns [29]. It is noteworthy that previous studies comparing plasma and serum did not find substantial differences in extracellular miRNA patterns [19]. Yet, differences between blood compartments may arise, e.g. upon platelet activation [19]. Finally, for qPCR-based analysis, utilization of two data normalization methods was deemed more trustworthy.

\section{Concentration of miRNA carriers improved detection of CRC tissue-related signatures}

Our results demonstrated that a simple polymerbased enrichment, prior to sensitive qPCR profiling, substantially improved detection of circulating miRNAs, thereby disclosing twofold relevant CRC-associated candidates that mirror deregulations in corresponding tissues. The recent work of Melo and colleagues [30] also showed that isolation of EVs was necessary to provide a more reliable (Glypican-1-based) diagnostic test for patients with pancreatic cancer, irrespective of the unclear intracellular origin of their marker [31]. Our results

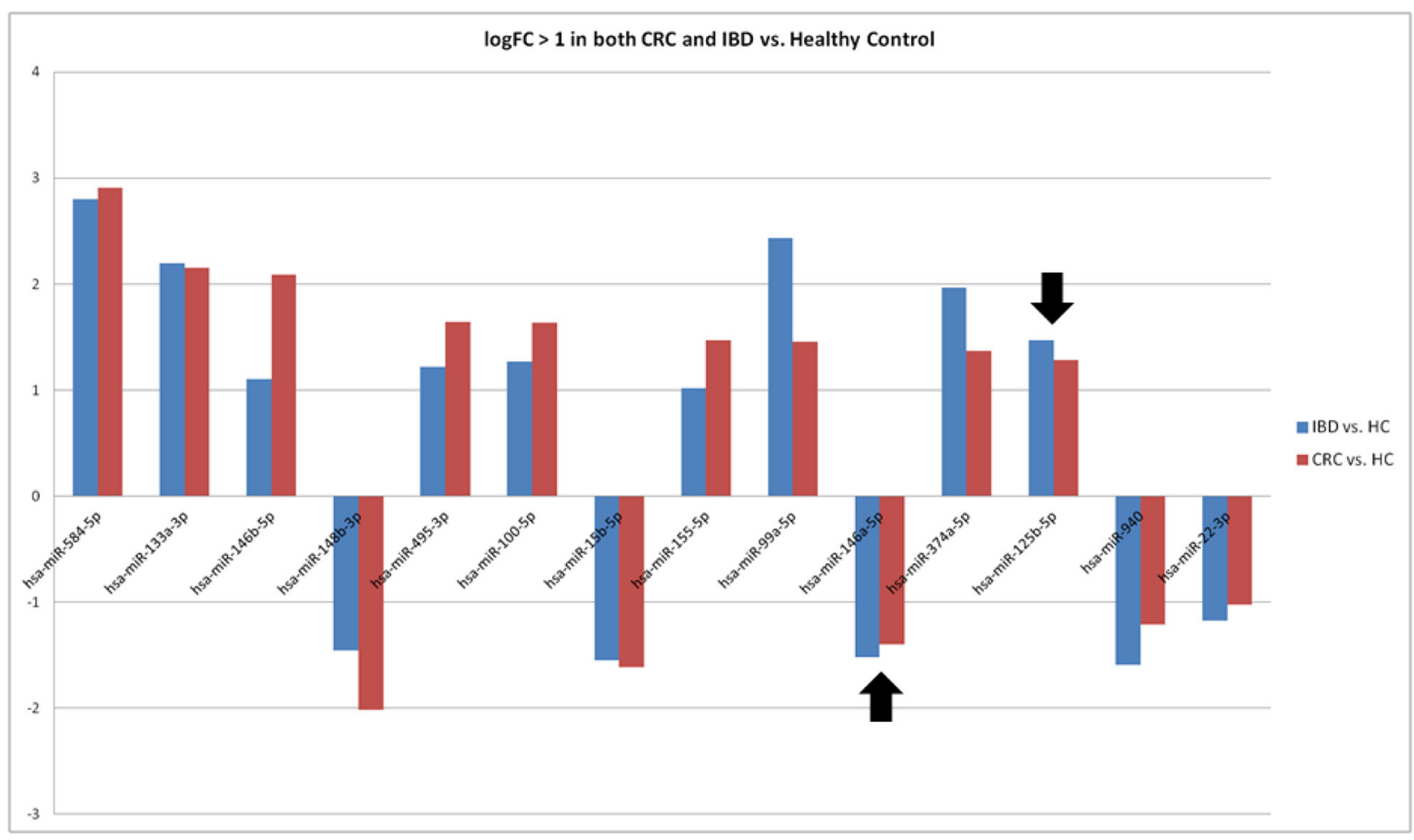

Figure 4: Consistent differential expression of 14 miRNAs in EV-Enriched sera fractions of both CRC and IBD. This figure demonstrates consistent overexpression of nine miRNAs and low expression of five miRNAs revealed in nuclease pre-treated, EV-enriched serum pools of CRC and IBD compared to a healthy control pool. The black arrows point to the elevated expression of miR$125 \mathrm{~b}-5 \mathrm{p}$ and reduced expression of miR-146a. 
are thus consistent with previous reports that highlight circulating EVs as stable [9], degradation-protected and enriched source [32] for miRNA biomarker discovery. The latter sequencing-based study reported 40 miRNAs that were confined inside serum EVs and were not detectable in serum and (PAXgene) blood. Similarly, we found a large set $(15 / 28 ;>53 \%)$ of differentially expressed miRNAs only in patients' particle-concentrated sera (see Supplementary Data). Furthermore, we found that all but one (miR-144) of the identified miRNAs were reported in the Extracellular Vesicles Database (http://microvesicles. org/). Remarkably, many of these candidates, such as miR15b, miR-21, miR-25, miR-92, miR-93, miR-223 and miR-486, can potentially be used as disease biomarkers in CRC [33].

Our results (Supplementary Tables S8 and S9) also suggest the possibility of crosstalk between these miRNAs and key inflammatory-/cancer-related factors, such as STAT3, and other STATs and cytokines [34]. For instance, miR-21, which was elevated in all CRC sera and tissues, has been reported to be increased in human colon tumors and correlated with tumor stage [35]. When transported by EVs, miR-21 can also induce tumor progression via pro-metastatic inflammatory responses by binding to Toll-like receptors and activation of NF- $\kappa \mathrm{B}-\mathrm{IL} 6 / \mathrm{TNF}-$ alpha signaling [36]. Another such example is miR-26a, which showed consistent elevation in sera and tissues of metastatic CRC patients (Supplementary Tables S4 and S10). Reported evidence indicated that overexpression of miR-26a can either reduce STAT3 phosphorylation in colon mucosa [37] or suppress tumor growth and metastasis through IL6-STAT3 signaling [38]. Yet, another study showed a reverse effect, where STAT3 can suppress miR-26a expression in T-cell lymphoma [39] (see Supplementary Discussion).

\section{Insights into the expression patterns of miR-486 and miR-92-a in CRC}

In the CRC field, the expression patterns of miR486 and miR-92a remain under debate. Their expression has been reported to be elevated in metastatic CRC with mutated KRAS, even prior to treatment [40]. Yet, a recent study [15] suggested that the reported elevation of both miRNAs in cancers (including CRC) mainly originated from erythrocytes, with a 20-30 fold increase in case of hemolysis. As hemolysis was absent or minimal in our serum samples, and given the consistent decrease of miR486 and miR-92a across all particle-concentrated sera (and whole sera in case of miR-486) and corresponding tissues (Table 1, Figure 3, and Supplementary Table S10), our results support the original hypothesis by Pritchard and colleagues [15]. In addition, it was reported [25] that the fluctuations of expression of both miRNAs are likely to rely on the EVs isolation method rather than different levels of $\mathrm{AGO} 2$ proteins bound to these
miRNAs, as previously suggested [5]. Therefore, the "down-regulation" signature of both miRNAs needs to be validated in larger independent cohorts.

\section{Evidence of strand-specific differential expression of miR-22 and miR-21 in CRC}

Our results suggest a possible "strand-specific" differential expression of extracellular miRNAs in CRC. For instance, both miR-22-3p and miR-22-5p were increased in paired $\mathrm{CRC}$ whole- and particle-concentrated sera, with a three-fold higher elevation of miR-22-5p than miR-22-3p in the respective enriched fractions than in whole sera (Table 1; Supplementary Table S3). Yet, a different scenario emerged in tumor tissues, which showed an elevation of miR-22-3p and down-regulation of miR22-5p. ANOVA analysis (Supplementary Table S7; Figure 3 ) showed more concordance of expression patterns of miR-22-3p in the matched cancer particle-concentrated sera and tissues. These expression differences suggest an oncogenic role of miR-22-3p and a tumor suppressor function of $\mathrm{miR}-22-5 \mathrm{p}$. Indeed, more relations to diseases (27 [including CRC] vs. two [hepatitis-B, prostate cancer]) and potential target genes (430 vs. 186) have been documented for miR-22-3p than of miR-22-5p (miRSearch v3: www.exiqon.com/miRSearch). miR-22 is known as a tumor suppressor in lung [41] and colon cancer [42], but it is unclear which mature form is most relevant. Down-regulation of miR-22 has been shown in CRC tissue and correlated with liver metastasis [43]. Deregulation of miR-22 has also been observed in tissues with IBD [44]. Remarkably, miR-22 is not expressed by blood cells [15]. Altogether, these data suggest that the abundance of miR-22 in the circulation may originate from the diseased (colon) tissue. The second candidate is miR-21. While miR-21-5p was overexpressed in CRC particle-concentrated sera and tissues, miR-21-3p was not detectable (Table 1; Figure 3). Previous studies also showed association of miR-21-5p expression with 134 diseases, including CRC (miRSearch v3).

To our knowledge, few studies have addressed this strand-specific miRNA-expression tendency. Examples are miR-28, miR-125, and miR-34c in CRC [45], lung [46] and cervical cancers [47], respectively. Nevertheless, in agreement with our results, a recent transcriptome analysis revealed a high abundance of both miR-22-3p and miR21-5p (two of five most overexpressed miRNAs) in a cohort of 88 CRC tumors [48].

\section{Implication of extracellular miRNAs in CRC and IBD}

Out of the identified 14 miRNAs, with consistent patterns of regulation in both neoplastic and inflammatory bowel diseases, the expression trends of both miR-125b$5 p$ and miR-146a-5p were in agreement with our first 
results. Both of these miRNAs are well known to be implicated in the regulation of immune functions and in CRC and IBD [49-52] (Supplementary Table S9). An additional candidate is miR-30b-5p, which was the only significantly overexpressed miRNA in CRC compared to IBD and the healthy individual serum pools. miR-30b-5p has been reported as a potential prognostic marker that mainly functions as a tumor suppressor [53] and mediator of metastatic and invasion behaviors in $\mathrm{CRC}[54,55]$. The higher expression of miR-30b in the "vesicle-enriched" CRC sera may indicate that tumor discards undesired inhibitory factors into the circulation via EVs. A higher expression of miR-30b in the circulation of CRC patients, compared to the corresponding $\mathrm{CRC}$ tissue and healthy controls (ANOVA, P-value: 0.0022), was also observed in our study (Supplementary Table S7). Taken together, the elevated expression level of miR-30b may suggest its utilization as an indicator for CRC progression.

In summary, enrichment of miRNA-containing particles from serum is evolving as a promising strategy for identifying cancer tissue-related miRNA signatures in liquid biopsies. As the clinical relevance and function of most of the known miRNAs are still undetermined, further studies are warranted to explore their specific association with key cellular pathways and inflammation-driven colon carcinogenesis, as well as possible biological implications of strand-specific expression. This may in turn provide insights into the pathogenesis of bowel diseases and lead to more effective therapies.

\section{MATERIALS AND METHODS}

\section{Subjects}

For this study a total number of 71 serum and 25 tissue samples were investigated. In the initial discovery and validation experiments, 29 serum samples, obtained from 19 clinical patients suffering from CRC and from 10 healthy individuals, as well as the corresponding CRC tissue samples $(n=25)$ from the resected tumors were analyzed (details in Supplementary Table S2). The 93 isolated RNAs from these samples were subjected to miRNA qPCR expression profiling (Supplementary Data). For a second independent validation approach, we analysed 42 additional serum samples (seven pools; six samples per pool) of cohorts suffering from CRC (UICC stage II disease; 18 samples in three pools), as well as ulcerative colitis (UC; 18 sera in three pools) and healthy individuals (six sera in one pool) (Supplementary Table S2c). The serum and tissue samples were obtained from the local oncological biomaterial bank of the Comprehensive Cancer Center North, University Clinic Schleswig-Holstein, Campus Kiel, and the Interdisciplinary Center for Biobanking-Lübeck (ICB-L). The sample collection and the analyses in this study were approved by the local review board and all donors gave informed written consent. Sera were prepared by centrifugation (2000 g, $10 \mathrm{~min}$ ) of blood samples, which had been either collected from healthy donors and patients with UC, by aspiration from the medial cubital vein or were collected from CRC patients prior to surgical tumor resection from a central venous line under general anesthesia. The tumor tissue specimens from patients being operated at the Department of General Surgery, University Clinic Schleswig-Holstein, Campus Kiel and were snap-frozen in liquid nitrogen $(n=15)$ or RNA stabilized by RNAlater reagent $(n=10$; Qiagen, Hilden, Germany). All samples were stored in the biobanks at minimum $-80^{\circ} \mathrm{C}$ until use.

\section{Preparation of particle-concentrated and particle-depleted serum fractions}

For enrichment of miRNA-containing particles, ExoQuick (SBI/BioCat, Heidelberg, Germany) was used following the manufacturer's instructions. Good performance of this isolation system has been reported previously [25]. To pre-evaluate the appropriate serum volume for particle-enrichment and RNA preparation, isolated particles from different volumes of serum were resuspended in $200 \mu \mathrm{l}$ Dulbecco's phosphate-buffered saline (PBS; Life Technologies, Darmstadt, Germany) and the total RNA was isolated (Qiagen miRNeasy kit). Subsequent analysis of yield and quality was performed by a Bioanalyzer 2100 (Agilent, Böblingen, Germany) using a RNA6000 Pico-kit (Agilent). Based on these experiments (data not shown), and as a compromise between RNA yield and input volume of clinical sera, $2 \mathrm{ml}$ serum samples were used for enriching the circulating miRNA-containing particles. Thawed sera were pre-cleared by centrifugation at $13.000 \mathrm{xg}$ for $10 \mathrm{~min}$ at $4^{\circ} \mathrm{C}$ and were mixed with $0.5 \mathrm{ml}$ ExoQuick reagent, incubated overnight at $4^{\circ} \mathrm{C}$ for particle precipitation, and subsequently centrifuged at 1,500 $\mathrm{xg}$ for $30 \mathrm{~min}$ at $4^{\circ} \mathrm{C}$. The pellets (particle-concentrated fractions) were resuspended in $1 / 10^{\text {th }}$ of the original serum volume, according to the manufacturer's protocol, i.e. in $200 \mu \mathrm{l} \mathrm{PBS}$. The supernatants were kept as particledepleted fractions. Nanoparticles tracking analysis (NTA) by a Nanosight NS300 (Malvern GmbH, Herrenberg, Germany) was used to assess size and concentration of isolated particles. Additionally, particle characteristics after isolation by ExoQuick were compared to two other isolation methods and after different storage conditions (Supplementary Figures S6 and S7).

\section{RNA isolation and quality checks}

Total RNA including miRNA was extracted from whole serum, and the resuspended particle-concentrated and particle-depleted sera, using the miRNeasy kit 
(Qiagen). Briefly, $200 \mu \mathrm{l}$ of each fraction (unprocessed serum, particle suspension and particle-depleted serum) was homogenized in $1 \mathrm{ml}$ Qiasol (Qiagen) and processed according to the manufacturer's instructions. Similarly, RNA from tissue samples $(30-60 \mathrm{mg}$ ) was extracted using the same kit after homogenization in Qiasol with a Precellys 24 homogenizer and ceramic beads $(1.4 \mathrm{~mm}$; PEQLAB, Erlangen, Germany). The RNA was stored at $-80^{\circ} \mathrm{C}$. Quality controls were undertaken to evaluate preanalytical and technical imbalances (Supplementary Data).

\section{cDNA synthesis and qPCR}

The RNA samples included in this study were reverse-transcribed into cDNA and run on miRCURY LNA $^{\mathrm{TM}}$ RT-miRNA-PCR panels (Exiqon, Vedbaek, Denmark). The Universal RT miRNA PCR Human panel-Iand-II (v2), consisting of 742 miRNA assays, was used in the first screen (30 RNA samples isolated from 10 matched CRC samples as whole sera, particle-concentrated and particle-depleted sera). The second panel, Universal $R T$ miRNA PCR Custom Pick\&Mix, consisting of 47 miRNA assays, was used for the subsequent experiments with the other 63 RNA samples (Figure 1). Moreover, as a third panel, a miRCURY LNA ${ }^{T M}$ Universal RT miRNA PCR Human panel $I$, which consists of 372 miRNAs, was used to independently analyze the miRNA expression patterns in three entities: CRC, ulcerative colitis (UC), and healthy volunteers (details in Supplementary Table S2c). Three pools of patient's sera for each disease, as well as one serum pool of healthy controls (6 individuals per pool; a total of 42 sera), were investigated. This third miRNApanel included most of the 45 miRNAs (identified in the initial screen; except for miR-320b, miR-1972, and miR-17-5p) and importantly, all but one (miR-320b) of the 22 miRNAs identified as differentially expressed in the particle-concentrated sera between $\mathrm{CRC}$ and healthy individuals (Figure 2). In this additional experiment, all seven serum pools $(0.5 \mathrm{ml}$ each $)$ were treated with DNase and RNase prior to isolation of miRNA-containing particles to digest extraneous nucleic acids. Briefly, 0.5 $\mathrm{ml}$ pooled sera was mixed with $50 \mu 110 \times$ DNase-reaction buffer (Promega, Heidelberg, Germany, \#M198A), $3 \mu \mathrm{l}$ DNase (Promega \#M6101), and $5 \mu \mathrm{l}$ RNase A (SigmaAldrich, Munich, Germany, \#R6148) and incubated for 60 min at RT. Then, $40 \mu \mathrm{l}$ of stop solution (Promega M199A) and $10 \mu \mathrm{l}$ (200 units) recombinant RNase inhibitor (ABI/ Thermo Fisher) were added. Next, total RNA, including miRNA, from exosomes and other EVs, was isolated using exoRNeasy method (Qiagen) according to the manufacturer's instructions.

\section{Data filtering and analysis}

The amplification efficiencies in the qPCRs were analyzed using algorithms similar to the LinReg software
(Supplementary Data). To improve our miRNA-selection, two data normalization methods were independently applied to the raw data of the initial screen (details in Supplementary Data). The overlapping miRNAs from both analyses, which showed statistically significant differential expression between the matched whole and particleconcentrated sera, were considered for follow up and in the combined downstream analyses (Figure 2). To assess miRNA expression differences between samples, fold changes were calculated, as well as, t-tests and multiple testing corrections according to the Benjamini-Hochberg method were applied. Furthermore, the miEAA tool (http://www.ccb.uni-saarland.de/mieaa_tool) was used to perform different overrepresentations and enrichment analyses (Supplementary Data).

\section{Abbreviations}

miRNA: microRNA; Cont: control; CRC: colorectal cancer; EVs: extracellular microvesicles; M1: CRC patients with distant metastasis; M0: CRC patients without metastasis; Ser: serum; NTA: Nanoparticle Tracking Analysis; IBD: inflammatory bowel disease; UC: ulcerative colitis; RT: room temperature.

\section{ACKNOWLEDGMENTS}

We thank all individuals for their participation, Michaela Schwarz, Melanie Friskovec, Catharina von der Lancken for technical assistance, and Søren Nielsen, Michael Thorsen for helping in data analysis.

\section{CONFLICTS OF INTEREST}

The authors declare that they have no competing interests.

\section{FUNDING}

This study was supported by the Deutsche Forschungsgemeinschaft (DFG), Cluster-of-Excellence 'Inflammation-at-Interfaces', the E:med project SysINFLAME CP4, and initially by Intramural-ResearchFunding of the Medical Faculty at Kiel University (CAU). Samples were supplied by the Kiel CCC-biomaterialbank, funded by the BMBF (PopGen 2.0 Network/ P2N-01EY1103), and the Interdisciplinary Center for Biobanking-Lübeck (ICB-L).

\section{Ethics and consent}

All participants gave written informed consent prior to participation. The project was approved by the Ethics Committee of the Medical Faculty of the ChristianAlbrechts-University of Kiel, Germany. 


\section{REFERENCES}

1. Keller A, Leidinger P, Bauer A, Elsharawy A, Haas J, Backes C, Wendschlag A, Giese N, Tjaden C, Ott K, Werner J, Hackert T, Ruprecht K, et al. Toward the bloodborne miRNome of human diseases. Nat Methods. 2011; 8:841-843.

2. Raisch J, Darfeuille-Michaud A and Nguyen HT. Role of microRNAs in the immune system, inflammation and cancer. World J Gastroenterol. 2013; 19:2985-2996.

3. Zhang HG and Grizzle WE. Exosomes: a novel pathway of local and distant intercellular communication that facilitates the growth and metastasis of neoplastic lesions. Am J Pathol. 2014; 184:28-41.

4. Fabbri M, Paone A, Calore F, Galli R, Gaudio E, Santhanam R, Lovat F, Fadda P, Mao C, Nuovo GJ, Zanesi N, Crawford M, Ozer GH, et al. MicroRNAs bind to Toll-like receptors to induce prometastatic inflammatory response. Proc Natl Acad Sci U S A. 2012; 109:E2110-2116.

5. Arroyo JD, Chevillet JR, Kroh EM, Ruf IK, Pritchard CC, Gibson DF, Mitchell PS, Bennett CF, Pogosova-Agadjanyan EL, Stirewalt DL, Tait JF and Tewari M. Argonaute2 complexes carry a population of circulating microRNAs independent of vesicles in human plasma. Proc Natl Acad Sci U S A. 2011; 108:5003-5008.

6. Vickers KC, Palmisano BT, Shoucri BM, Shamburek RD and Remaley AT. MicroRNAs are transported in plasma and delivered to recipient cells by high-density lipoproteins. Nat Cell Biol. 2011; 13:423-433.

7. Turchinovich A, Weiz L and Burwinkel B. Isolation of circulating microRNA associated with RNA-binding protein. Methods Mol Biol. 2013; 1024:97-107.

8. Gallo A, Tandon M, Alevizos I and Illei GG. The majority of microRNAs detectable in serum and saliva is concentrated in exosomes. PLoS One. 2012; 7:e30679.

9. Ge Q, Zhou Y, Lu J, Bai Y, Xie X and Lu Z. miRNA in Plasma Exosome is Stable under Different Storage Conditions. Molecules. 2014; 19:1568-1575.

10. Rodriguez M, Silva J, Herrera A, Herrera M, Pena C, Martin P, Gil-Calderon B, Larriba MJ, Coronado MJ, Soldevilla B, Turrion VS, Provencio M, Sanchez A, Bonilla F and GarciaBarberan V. Exosomes enriched in stemness/metastaticrelated mRNAS promote oncogenic potential in breast cancer. Oncotarget. 2015; 6:40575-40587. doi: 10.18632/ oncotarget.5818.

11. Yu S, Cao H, Shen B and Feng J. Tumor-derived exosomes in cancer progression and treatment failure. Oncotarget. 2015; 6:37151-37168. doi: 10.18632/oncotarget.6022.

12. Raposo G and Stoorvogel W. Extracellular vesicles: exosomes, microvesicles, and friends. J Cell Biol. 2013; 200:373-383.

13. Yong FL, Law CW and Wang CW. Potentiality of a triple microRNA classifier: miR-193a-3p, miR-23a and miR$338-5 p$ for early detection of colorectal cancer. BMC Cancer. 2013; 13:280.
14. Keller A, Leidinger P, Vogel B, Backes C, ElSharawy A, Galata V, Mueller SC, Marquart S, Schrauder MG, Strick R, Bauer A, Wischhusen J, Beier M, et al. miRNAs can be generally associated with human pathologies as exemplified for miR-144. BMC Med. 2014; 12:224.

15. Pritchard CC, Kroh E, Wood B, Arroyo JD, Dougherty KJ, Miyaji MM, Tait JF and Tewari M. Blood cell origin of circulating microRNAs: a cautionary note for cancer biomarker studies. Cancer Prev Res (Phila). 2012; 5:492-497.

16. Turchinovich A and Cho WC. The origin, function and diagnostic potential of extracellular microRNA in human body fluids. Front Genet. 2014; 5:30.

17. Volinia S, Calin GA, Liu CG, Ambs S, Cimmino A, Petrocca F, Visone R, Iorio M, Roldo C, Ferracin M, Prueitt RL, Yanaihara N, Lanza G, et al. A microRNA expression signature of human solid tumors defines cancer gene targets. Proc Natl Acad Sci U S A. 2006; 103:2257-2261.

18. Witwer KW. Circulating MicroRNA Biomarker Studies: Pitfalls and Potential Solutions. Clin Chem. 2015; 61:56-63.

19. Moldovan L, Batte KE, Trgovcich J, Wisler J, Marsh $\mathrm{CB}$ and Piper M. Methodological challenges in utilizing miRNAs as circulating biomarkers. J Cell Mol Med. 2014; 18:371-390.

20. Yang $\mathrm{C}$ and Robbins PD. Immunosuppressive exosomes: a new approach for treating arthritis. Int J Rheumatol. 2012; 2012:573528.

21. Turchinovich A, Weiz L and Burwinkel B. Extracellular miRNAs: the mystery of their origin and function. Trends Biochem Sci. 2012; 37:460-465.

22. Chevillet JR, Kang Q, Ruf IK, Briggs HA, Vojtech LN, Hughes SM, Cheng HH, Arroyo JD, Meredith EK, Gallichotte EN, Pogosova-Agadjanyan EL, Morrissey C, Stirewalt DL, et al. Quantitative and stoichiometric analysis of the microRNA content of exosomes. Proc Natl Acad Sci U S A. 2014; 111:14888-14893.

23. Melo SA, Sugimoto H, O'Connell JT, Kato N, Villanueva A, Vidal A, Qiu L, Vitkin E, Perelman LT, Melo CA, Lucci A, Ivan C, Calin GA and Kalluri R. Cancer Exosomes Perform Cell-Independent MicroRNA Biogenesis and Promote Tumorigenesis. Cancer cell. 2014; 26:707-721.

24. Taylor DD, Zacharias W and Gercel-Taylor C. Exosome isolation for proteomic analyses and RNA profiling. Methods Mol Biol. 2011; 728:235-246.

25. Rekker K, Saare M, Roost AM, Kubo AL, Zarovni N, Chiesi A, Salumets A and Peters M. Comparison of serum exosome isolation methods for microRNA profiling. Clin Biochem. 2014; 47:135-138.

26. McKenzie AJ, Hoshino D, Hong NH, Cha DJ, Franklin JL, Coffey RJ, Patton JG and Weaver AM. KRAS-MEK Signaling Controls Ago2 Sorting into Exosomes. Cell Rep. 2016; 15:978-987.

27. Van Deun J, Mestdagh P, Sormunen R, Cocquyt V, Vermaelen K, Vandesompele J, Bracke M, De Wever O 
and Hendrix A. The impact of disparate isolation methods for extracellular vesicles on downstream RNA profiling. J Extracell Vesicles. 2014; 3.

28. Turchinovich A, Weiz L, Langheinz A and Burwinkel B. Characterization of extracellular circulating microRNA. Nucleic Acids Res. 2011; 39:7223-7233.

29. Cheng HH, Yi HS, Kim Y, Kroh EM, Chien JW, Eaton KD, Goodman MT, Tait JF, Tewari M and Pritchard CC. Plasma processing conditions substantially influence circulating microRNA biomarker levels. PLoS One. 2013; 8:e64795.

30. Melo SA, Luecke LB, Kahlert C, Fernandez AF, Gammon ST, Kaye J, LeBleu VS, Mittendorf EA, Weitz J, Rahbari N, Reissfelder C, Pilarsky C, Fraga MF, Piwnica-Worms D and Kalluri R. Glypican-1 identifies cancer exosomes and detects early pancreatic cancer. Nature. 2015; 523:177-182.

31. Thery C. Cancer: Diagnosis by extracellular vesicles. Nature. 2015; 523:161-162.

32. Cheng L, Sharples RA, Scicluna BJ and Hill AF. Exosomes provide a protective and enriched source of miRNA for biomarker profiling compared to intracellular and cell-free blood. J Extracell Vesicles. 2014; 3.

33. Revenfeld AL, Baek R, Nielsen MH, Stensballe A, Varming $\mathrm{K}$ and Jorgensen M. Diagnostic and prognostic potential of extracellular vesicles in peripheral blood. Clin Ther. 2014; 36:830-846.

34. Kohanbash $\mathrm{G}$ and Okada H. MicroRNAs and STAT interplay. Semin Cancer Biol. 2012; 22:70-75.

35. Bakirtzi K, Hatziapostolou M, Karagiannides I, Polytarchou C, Jaeger S, Iliopoulos D and Pothoulakis C. Neurotensin signaling activates microRNAs-21 and -155 and Akt, promotes tumor growth in mice, and is increased in human colon tumors. Gastroenterology. 2011; 141:1749-1761 e1741.

36. Fabbri M, Paone A, Calore F, Galli R and Croce CM. A new role for microRNAs, as ligands of Toll-like receptors. RNA Biol. 2013; 10:169-174.

37. Koukos G, Polytarchou C, Kaplan JL, Morley-Fletcher A, Gras-Miralles B, Kokkotou E, Baril-Dore M, Pothoulakis C, Winter HS and Iliopoulos D. MicroRNA-124 regulates STAT3 expression and is down-regulated in colon tissues of pediatric patients with ulcerative colitis. Gastroenterology. 2013; 145:842-852 e842.

38. Yang X, Liang L, Zhang XF, Jia HL, Qin Y, Zhu XC, Gao XM, Qiao P, Zheng Y, Sheng YY, Wei JW, Zhou HJ, Ren N, Ye QH, Dong QZ and Qin LX. MicroRNA-26a suppresses tumor growth and metastasis of human hepatocellular carcinoma by targeting interleukin-6-Stat3 pathway. Hepatology. 2013; 58:158-170.

39. Zhu H, Vishwamitra D, Curry CV, Manshouri R, Diao L, Khan A and Amin HM. NPM-ALK up-regulates iNOS expression through a STAT3/microRNA-26a-dependent mechanism. J Pathol. 2013; 230:82-94.

40. Ragusa M, Majorana A, Statello L, Maugeri M, Salito L, Barbagallo D, Guglielmino MR, Duro LR, Angelica
R, Caltabiano R, Biondi A, Di Vita M, Privitera G, et al. Specific alterations of microRNA transcriptome and global network structure in colorectal carcinoma after cetuximab treatment. Mol Cancer Ther. 2010; 9:3396-3409.

41. Ling B, Wang GX, Long G, Qiu JH and Hu ZL. Tumor suppressor miR-22 suppresses lung cancer cell progression through post-transcriptional regulation of ErbB3. J Cancer Res Clin Oncol. 2012; 138:1355-1361.

42. Li B, Song Y, Liu TJ, Cui YB, Jiang Y, Xie ZS and Xie SL. miRNA-22 suppresses colon cancer cell migration and invasion by inhibiting the expression of T-cell lymphoma invasion and metastasis 1 and matrix metalloproteinases 2 and 9. Oncol Rep. 2013; 29:1932-1938.

43. Zhang G, Xia S, Tian H, Liu Z and Zhou T. Clinical significance of miR-22 expression in patients with colorectal cancer. Med Oncol. 2012; 29:3108-3112.

44. Fasseu M, Treton X, Guichard C, Pedruzzi E, Cazals-Hatem D, Richard C, Aparicio T, Daniel F, Soule JC, Moreau R, Bouhnik Y, Laburthe M, Groyer A and Ogier-Denis E. Identification of restricted subsets of mature microRNA abnormally expressed in inactive colonic mucosa of patients with inflammatory bowel disease. PLoS One. 2010; 5.

45. Almeida MI, Nicoloso MS, Zeng L, Ivan C, Spizzo R, Gafa R, Xiao L, Zhang X, Vannini I, Fanini F, Fabbri M, Lanza G, Reis RM, Zweidler-McKay PA and Calin GA. Strand-specific miR-28-5p and miR-28-3p have distinct effects in colorectal cancer cells. Gastroenterology. 2012; 142:886-896 e889.

46. Jiang L, Huang Q, Zhang S, Zhang Q, Chang J, Qiu X and Wang E. Hsa-miR-125a-3p and hsa-miR-125a-5p are downregulated in non-small cell lung cancer and have inverse effects on invasion and migration of lung cancer cells. BMC Cancer. 2010; 10:318.

47. Lopez JA and Alvarez-Salas LM. Differential effects of miR-34c-3p and miR-34c-5p on SiHa cells proliferation apoptosis, migration and invasion. Biochem Biophys Res Commun. 2011; 409:513-519.

48. Schee K, Lorenz S, Worren MM, Gunther CC, Holden M, Hovig E, Fodstad O, Meza-Zepeda LA and Flatmark K. Deep Sequencing the MicroRNA Transcriptome in Colorectal Cancer. PLoS One. 2013; 8:e66165.

49. Liu J, Zheng M, Tang YL, Liang XH and Yang Q. MicroRNAs, an active and versatile group in cancers. Int $\mathrm{J}$ Oral Sci. 2011; 3:165-175.

50. Chen WX, Ren LH and Shi RH. Implication of miRNAs for inflammatory bowel disease treatment: Systematic review. World J Gastrointest Pathophysiol. 2014; 5:63-70.

51. Nishida N, Yokobori T, Mimori K, Sudo T, Tanaka F, Shibata K, Ishii H, Doki Y, Kuwano $\mathrm{H}$ and Mori M. MicroRNA miR-125b is a prognostic marker in human colorectal cancer. Int J Oncol. 2011; 38:1437-1443.

52. Tili E, Michaille JJ, Cimino A, Costinean S, Dumitru CD, Adair B, Fabbri M, Alder H, Liu CG, Calin GA and Croce CM. Modulation of miR-155 and miR-125b levels following lipopolysaccharide/TNF-alpha stimulation and 
their possible roles in regulating the response to endotoxin shock. J Immunol. 2007; 179:5082-5089.

53. Liao WT, Ye YP, Zhang NJ, Li TT, Wang SY, Cui YM, Qi L, Wu P, Jiao HL, Xie YJ, Zhang C, Wang JX and Ding YQ. MicroRNA-30b functions as a tumour suppressor in human colorectal cancer by targeting KRAS, PIK3CD and BCL2. J Pathol. 2014; 232:415-427.
54. Zhao H, Xu Z, Qin H, Gao Z and Gao L. miR-30b regulates migration and invasion of human colorectal cancer via SIX1. Biochem J. 2014; 460:117-125.

55. Park SY, Kim H, Yoon S, Bae JA, Choi SY, Jung YD and Kim KK. KITENIN-targeting microRNA-124 suppresses colorectal cancer cell motility and tumorigenesis. Mol Ther. 2014; 22:1653-1664. 